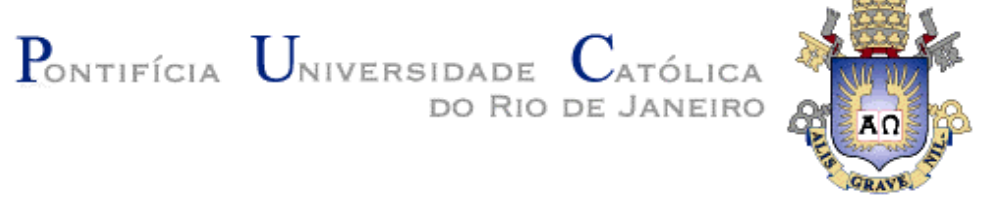

Marcelo Leta Vieira

\title{
Integração de Informação entre Cliente e Produtores Sob Contrato:
}

Estudo de caso.

\section{Dissertação de Mestrado}

(Opção profissional)

Dissertação apresentada como requisito parcial para obtenção do título de Mestre (opção profissional) pelo Programa de Pós-Graduação em Engenharia de Produção do Departamento de Engenharia Industrial da PUC-Rio.

Orientador: Prof. Leonardo Lustosa

Rio de Janeiro

Outubro de 2008 


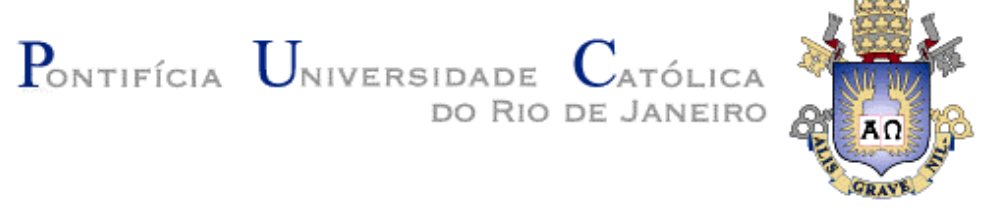

Marcelo Leta Vieira

\title{
Integração de Informação entre Cliente e Produtores sob Contrato:
}

Estudo de caso.

Dissertação apresentada como requisito parcial para obtenção do título de Mestre (opção profissional) pelo Programa de Pós-Graduação em Engenharia de Produção do Departamento de Engenharia Industrial da PUC-Rio. Aprovada pela Comissão Examinadora abaixo assinada.

\author{
Prof. Leonardo Junqueira Lustosa \\ Orientador \\ Departamento de Engenharia Industrial - PUC-Rio
}

Prof. Antônio Fernando de Castro Vieira Departamento de Engenharia Industrial - PUC-Rio

Prof. Nélio Domingues Pizzolato Departamento de Engenharia Industrial - PUC-Rio

Prof. José Eugênio Leal

Coordenador(a) Setorial do Centro Técnico Científico - PUC-Rio

Rio de Janeiro, 10 de outubro de 2008 
Todos os direitos reservados. É proibida a reprodução total ou parcial do trabalho sem autorização da universidade, do autor e do orientador.

\section{Marcelo Leta Vieira}

Graduou-se em Matemática aplicada à Informática (Faculdade Nuno Lisboa) em 1993. Pós-Graduado em Gestão de Negócios e Tecnologia da Informação (Fundação Getúlio Vargas) em 2002. Atuou como Analista de Negócios na empresa de Telecomunicações TIM, na Distribuidora de Cosméticos do grupo WELLA e na Indústria Química e Farmacêutica MERCK S.A. Atuou também como docente e coordenador de informática do curso técnico profissionalizante de Processamento de Dados do Colégio Santa Mônica. Atualmente é Gerente de Sistemas da CERAS JOHNSON, multinacional atuante no segmento de bens de consumo.

Ficha Catalográfica

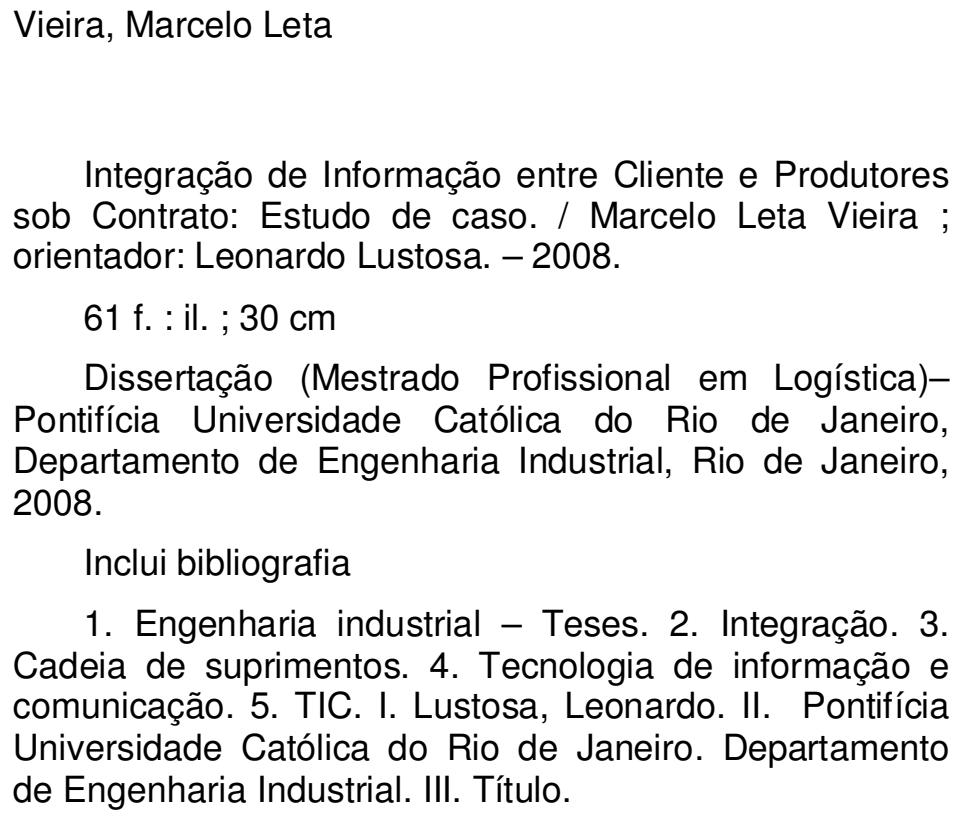

Dissertação (Mestrado Profissional em Logística)Pontifícia Universidade Católica do Rio de Janeiro, Departamento de Engenharia Industrial, Rio de Janeiro, 2008.

Inclui bibliografia

1. Engenharia industrial - Teses. 2. Integração. 3. Cadeia de suprimentos. 4. Tecnologia de informação e comunicação. 5. TIC. I. Lustosa, Leonardo. II. Pontifícia Universidade Católica do Rio de Janeiro. Departamento de Engenharia Industrial. III. Título.

CDD: 658.5 


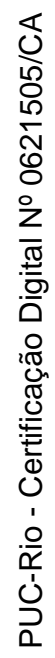

Para minha esposa e meus filhos: Geisa, Felipe e Pedro. 


\section{Agradecimentos}

A minha esposa, por todo seu esforço, apoio, compreensão e paciência. E aos meus filhos pela serenidade com a qual suportaram minha quase ausência nesses dois longos anos.

Aos meus pais, pela dedicação de suas vidas e aos meus irmãos, pela confiança e apoio de sempre.

Aos meus poucos, mas verdadeiros amigos, pelo apoio e pela torcida.

Ao professor Luis César (FGV) e aos colegas de trabalho Pedro Neves e André Santos, pela confiança ao colocarem seus nomes em meus formulários de recomendação para inscrição no curso.

Aos colegas de trabalho Aldo, Felipe, Joey e Rubens, por toda atenção e auxílio. Primordiais para compreensão detalhada dos processos da empresa.

Aos funcionários da biblioteca da PUC e do CCE, pela presteza e consideração.

Aos colegas de turma, pelo companheirismo.

Aos professores do curso, pelo brilhantismo de suas aulas e pelo respeito com o qual fui tratado.

Ao professor Leonardo Lustosa, que me deu a honra de sua orientação, pela maneira como fui acolhido, pela dedicação de seu tempo e por toda atenção e respeito que me foram dispensados.

Por fim, graças a Deus. 


\section{Resumo}

Vieira, Marcelo Leta. Integração de Informação entre Cliente e Produtores sob Contrato: Estudo de Caso. Rio de Janeiro, 2008. 61p. Dissertação de Mestrado (Opção profissional) - Departamento de Engenharia Industrial, Pontifícia Universidade Católica do Rio de Janeiro.

Não há duvidas acerca do fato de que as Tecnologias de Informação e Comunicação (TIC) se tornaram uma poderosa ferramenta para viabilizar a integração e o gerenciamento das cadeias de suprimentos (SCM). No cenário atual das grandes organizações multinacionais, que competem em mercados globalizados, não é possível pensar em integração sem o apoio de tais ferramentas. Entretanto, mesmo com os avanços tecnológicos, ainda é possível observar projetos que envolvem cadeias de suprimento sem o apoio adequado de TIC. Este trabalho é um estudo de caso que aborda o relacionamento entre TIC e SCM sob o ponto de vista das implicações nos processos de negócios. Ele toma como base a subsidiária brasileira de uma empresa multinacional de grande porte e seus fornecedores diretos no Brasil. O estudo evidencia os problemas decorrentes da operação com os terceiros sem o apoio adequado de TIC, por exemplo, o aumento da carga de trabalho em diferentes setores da empresa. Além disso, são apontados os potenciais riscos para a companhia, como o aumento nos níveis de inventário, ou nos custos operacionais, e a queda no nível de serviço ao cliente. Também são descritos os planos de médio e longo prazo da empresa para a resolução do problema e apresentadas sugestões de ações de curto prazo que podem minimizar os problemas encontrados e reduzir os riscos dessa operação a um nível seguro.

\section{Palavras-chave}

Integração; Cadeia de Suprimentos; Tecnologia de Informação e Comunicação; TIC. 


\section{Abstract}

Vieira, Marcelo Leta. Integration of Information between Customer and Contract Manufacturers: Case study. Rio de Janeiro, 2008. 61p. MSc Dissertation - Departamento de Engenharia Industrial, Pontifícia Universidade Católica do Rio de Janeiro.

There is no doubt concerning the fact that Information Technology (IT) has become a powerful tool to make possible the integration and the Supply Chain Management (SCM). In the current scene of the multinational companies, which compete in global markets, it is not possible to think about integration without the support of such tools. However, even with the technological advances it is still possible to observe projects involving supply chain without adequate IT support. This research is a case study about the relationship between IT and SCM, under the view of the business processes implications. It takes as base the Brazilian subsidiary of a large multinational company and their main direct vendors in Brazil. The study shows the problems generated by the operation with third partners without adequate IT support, for example, the work load increase in different sectors of the company. Moreover, the potential risks to the company are pointed out, as the increase in the inventory level or in the operational costs, and the customer service level decrease. The medium and long-term plans of the company to solve the problems are also described, and suggestions are given for short-term actions that can minimize the problems listed and reduce the operational risk to a safe level.

\section{Keywords}

Integration; Supply Chain; Information Technology; SCM; IT. 


\section{Sumário}

1 Introdução 12

1.1. O Problema 13

1.2. Objetivos 13

1.3. Relevância do Estudo 14

1.4. Delimitação do estudo 14

1.5. Metodologia 15

2 Referencial teórico 16

2.1. Conceitos e definições 16

2.2. Importância da informação e da TIC nos processos e no gerenciamento da cadeia de suprimentos 19

2.3. A evolução da TIC aplicada à cadeia de suprimentos 20

2.4. Planejamento Colaborativo, Previsão e Reabastecimento 24

3 Estudo de caso 28

3.1. Histórico da empresa 28

3.2. A operação da empresa no Brasil 30

3.3. O desafio da operação com terceiros 31

3.4. A motivação para o estudo de caso 32

3.5. Resumo dos processos 34

3.5.1. O processo de S\&OP (Sales and Operative Plan): 36

3.5.2. A interação entre a SCJ e os terceiros: 38

3.6. O Diagnóstico do problema 40

3.7. As consequências do problema 45

3.8. A Solução proposta 46

$\begin{array}{ll}\text { 3.9. Resultados esperados } & 49\end{array}$

4 Conclusões $\quad 51$

4.1. Resposta ao objetivo principal do estudo 51

4.2. Resposta ao objetivo acessório do estudo 52 
4.3. Considerações finais

53

4.4. Sugestões para pesquisas futuras

55

4.4.1. A escolha dos fornecedores

55

4.4.2. A definição do escopo da integração

57

5 Referências bibliográficas

59

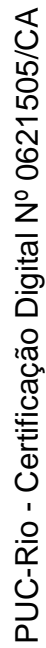




\section{Lista de figuras}

Figura 1: Evolução dos sistemas até o ERP.

Figura 2: Evolução da abordagem de desenvolvimento das cadeias de suprimento.

Figura 3: Fluxo da operação brasileira da SCJ antes da reestruturação. 30 Figura 4: Fluxo da operação brasileira da SCJ após a reestruturação. 31 Figura 5: Faturamento bruto por origem da produção (Planejamento 2008).

Figura 6: Localização dos Fornecedores. 35

Figura 7: Resumo do processo de S\&OP. 36

Figura 8: Fluxo do processo de S\&OP. 38

Figura 9: Fluxo da interação entre a SCJ e os terceiros. 